\title{
Exploring Role of Adipose Organ and Dysmetabolic Effects of Adiposity: Over-Nutrition, Nutrient Overload, Insulin Resistance, T2DM and Other Fallouts
}

\author{
Vinod Nikhra* \\ Department of Medicine, India \\ *Corresponding author: Vinod Nikhra, Senior Chief Medical Officer and Consultant, Department of Medicine, H New Delhi, India
}

Submission: 眥 May 02, 2018; Published: 制July 11, 2018

\begin{abstract}
The complex adipose organ

It has highly plasticproperties to reprogram its genes and transdifferentiate reversibly into cells with different structure and functions. The adipose organ plays important role in health and disease states including obesity and MetS and fulfils several crucial survival needs like thermogenesis, storage of energy and fuel for metabolism, immune responses, sexual development around puberty, reproductive function and lactation. Further, as an endocrine organ, it secretes several peptides termed adipokines, including adiponectin and leptin.
\end{abstract}

\section{Adiposity and metabolic mechanisms}

The rising prevalence of obesity and MetS in recent decades reflects increased calorie consumption though diets rich in fat and refinedcarbohydrates, leading to over-nutrition and nutrient overload which in due course leads to nutritional toxicity, endangering the intracellular organelle and impairing intracellular and intercellular metabolic processes. The adipose tissue protects the vital organs through expansion of the WAT, through an upsurge in the adipocytecellsize and formation of new white adipocytes, and adiposity can, thus, be regarded a way to deal with nutritional excess.The IR linked to adiposity can also be regarded a measure to control excess nutrients entry into cells, endangering vital intracellular organs.

\section{The excess folic acid and obesity link}

Folic acid influences energy aswell as lipid metabolism by modifying DNA synthesis and gene expressions and contributes to alterations in metabolism. In the animal model, the EFA with a HF diet were related to significant weight and fat mass gain. Thus, EFA may intensify weight increase, adiposity and inflammatory response in setting of increased fat dietary intake.

\section{Metabolic fallouts of adiposity/obesity}

The adipose tissue relates to about $20 \%$ of body weight in lean individuals and to $50 \%$ or more body weight in extremely obese. The adipose tissue organ has white, beige and brown adipocytes for specialized functions, and their coordinated action ensure an optimal metabolic homeostasis. The adiposity is a crucial factor in development of metabolic diseases including DM, and high BMI, T2DM and IR are closely linked.With obesity, the levels of NEFAs, adiponectins, cytokines and proinflammatory markers like NNF- $\alpha$ and IL-6 involved in the genesis of IR, are increased, and with metabolic dysfunctions, there occur derangements of carbo-lipid and global metabolic homeostasis. Normally, there is a dynamic feedback relationship between $\beta$-cells function and insulin-sensitive response in tissues. The failure of the intricate process and sustained decline in $\beta$-cell function result in dysregulation of glucose levels and worsening ofT2DM.

\section{Future projections and options}

Both obesity and T2DM represent a global public health crisis brought about by rapid westernization, nutritional alterations transition and increasingly sedentary lifestyles. Controlling and treating Obesity as well as T2DM and their fallouts require a thoughtful long-term planning and rational use of limitedresourcesin developing countries with scarce resources. Further, owing to the wide range of therapeutic interventions and options available, the treatment algorithm is ridden with complexity.

Keywords:Adiposity; Obesity; Adipose tissue; Metabolic syndrome; T2DM; Insulin resistance; Folic acid

Abbreviations: AD: Alzheimer's Disease; AdipoQ: Adiponectin or Adipocyte Complement-Related Protein; AdipoQ gene: APM1, Mapped to Chromosome 3q27; AFA: Adequate Folic Acid; AMPK: Activated Mono Protein Kinase; ATP: Adenosine Tri Phosphate; BAT: Brown Adipose Tissue; BFD: Body Fat Distribution; BLAs: Brown-Like Adipocytes; BMI: Body Mass Index; $\beta$-cells: $\beta$-cells of Islets of Langerhans; $\beta$-3 Ars:Beta3 Adrenoreceptors; $11 \beta H S D-1$ : 11 $\beta$-Hydroxy Steroid Dehydrogenase Type 1; CLSs: Crown Like Structures; CNS: Central Nervous System; COX2: Cyclooxygenase 2; CVD: Cardio Vascular Disease; cAMP: Cyclic Adeno Mono Phosphate; DM: Diabetes Mellitus; EFA: Excess Folic Acid; ERK: Extracellular Signal Regulated Kinase; FFA: Free Fatty Acids; FGF21: Growth Factor; FOXC2: Fork Head Box Protein C2; HF-EFA:High Fat-Excess Folic Acid; IL-1 $\alpha$ : Interleukin1 $\alpha$, IL-6: Interleukin-6; IR: Insulin Resistance; LPS: Lipo Poly Saccharide; MetS: Metabolic Syndrome; mRNA: Messenger-RNA; MHO: Metabolically Healthy Obese; PPAR $\gamma$ :Peroxisome Proliferator Activated Receptor- $\gamma$; Rb protein: Retinoblastoma Protein; SF:Subcutaneous Fat; ss3AR: Specific Adeno Receptor; S100-B: S100 Calcium Binding Protein B; SOCS-3: Suppressorof Cytokine Signaling; Smad3: Component of the TGFß signaling pathway; TGFß: Transforming Growth Factor Beta; T1DM: Type 1 Diabetes Mellitus; T2DM: Type 2 Diabetes NMellitus; TH:Tyrosine Hydroxylase;TNF- $\alpha$ :Tumour Necrosis Factor Alfa;TZDs: Thiazolidinediones; UCP1: Uncoupling Protein 1; VF: Visceral Fat; WAT: White Adipose Tissue 


\section{Adipose Tissue: The Complex Organ}

The adipose tissue is a complex organ with characteristicsfor a high degree of plasticity anda unique property of the adipocytes to be able to reprogram their genes totransdifferentiatereversibly into cells with a different structure and functions. As documented by various recent studies, highlighting its active physiological role in various stages of life including puberty, pregnancy and lactation,the adipose tissue as a wholecan aptly be referred to as 'adipose organ' [1].The adipose organ plays important roles both in health as well as disease states including obesity and metabolic syndrome (MetS).

\section{The adipose tissue cytology}

The adipose organ is composed of two main types of adipocytes, the white adipose tissue (WAT)which are spherical cells with $\sim 90 \%$ of their volume comprising a single large cytoplasmic lipid droplet and an enfolded nucleus and is leptin-responsive and S100B-immunoreactive. The brown adipose tissue (BAT) consistsof polygonal cells with a round nucleus and have and numerous cytoplasmic lipid droplets. The BAT is rich in mitochondria with well-defined cristae anddistinctly express the uncoupling protein 1 (UCP1), a unique protein responsible for oxidative phosphorylation for thermogenesis. Both, the WAT and BATexist in various depots of the adipose organ, consisting of subcutaneous(mainly large adipocytes) and visceral depots (mainly small adipocytes). Some of adipose depots areas are brown, that is, consist mainly of brown adipose tissue, while the white adipose areas consistof a majority of white adipose tissue and contain also a variable number of brown adipocytes, the number of which varies with age, metabolic state and environmental factors.

\section{The adipose tissue depots}

The adipose organ fulfils to organism's some of the key survival needs like thermogenesis, stores for metabolic fuel and energy, immune responsiveness,sexual development around puberty, reproductive function and lactation. The white adipocytes stores energy and nutrients, whereas the brown adipocytesutilize energy for thermogenesis to maintain body temperature. The two types of adipose cells with opposing functions co-exist in various fat depots of adipose organ, a remarkable feature which is critical for the adipose organ's plasticity [2].The adipose organ is rich in blood vessels and nerve fibres, their density being comparatively higher in the brown zones. All the adipocytes from the adipose organ have been shown to express the specific adrenoceptor (ss3AR), which is related, to the plasticity and in case of the loss of ss3ARs the plasticity of adipose tissue is decreased [3].

The adipose tissue is made up of multiplesubcutaneous and visceral zones. The anterior subcutaneous zone - main volume is located at upper dorsal area at level of the scapulae consisting of inter-scapular, subscapular, axillary and cervical. The posterior subcutaneous zone, located mainly in lower ventral part of body and consists of dorso-lumbar, inguinal and gluteal depots. The truncal adipose zone is contained in mediastinum and abdomen. Apart from these, in females, the abdominopelvic adipose zone consists of perirenal, periovarian, parametrial and perivesicaldepots.

\section{The composition of adipose organ}

The composition of adipose tissueisvariabledepending upon the location,metabolic state and environmental conditions. In the hot weather, adipose zones near the aorta and arteries, inter-scapular region, several subcutaneous and visceral regions represent BAT,containing mainly UCP1-expressing brown adipocytes, highlyvascular network and richly innervating noradrenergic parenchymal fibers. Whereas, majority of the adipose organ, represents WAT, composed ofleptinand S100Bexpressing adipose tissue which is deficiently vascularized and innervated. Some areas are composed of paucilocular adipocytes positive for UCP1 and S100-B, depending on their differentiation stage, and are intermediate between white and brown adipocytes, and called beige or brite adipocytes. The beige cellshave a gene expression pattern distinct from that of either white or brown fat cells,resembling white fat cells in the basal state, but respond to environmental thermogenic stimuli leading to increase in thermogenic genes [4]. Thus, the adipose tissues as an organ with discrete anatomy, specific vascular and nerve supplies, has a complex cytologyandhighplasticity.

Thus, both white and brown adipocytes are present together in visceral and subcutaneous zones, though in a variable proportion,in spite of their different functions of storing energy and producing heat respectively. It is apparent that such locational arrangement is directly related to their plasticity, which following change in milieu and apt stimulation letstrans-differentiation of an adipocyte type into the otherone [5].Undersituations of a continuous and chronic cold exposure, white-to-brown transdifferentiation occurs to meet the increased need for thermogenesis, whereas a regular intake of obesogenic diet induces brown-to-white conversion to fulfill the increased requirementto deal with thenutrient overload. T2DM is the most common metabolic disease associated withcentral obesity. With adiposity comes the deficient oxygenation and adipocytes undergo apoptosis, initiating macrophages infiltration of the adipose tissue bringing about a low-grade chronic inflammation and release of proinflammatoryfactorspaving to IR and T2DM, in due course.

\section{Dynamics of adipose organ}

The pericardial, omental, mesenteric and subcutaneous adipose depots normally contain lymphocytes. But, the functional relationship between adipocytes and lymphocytes is altered in adiposity.In general, the brown adipocytes are smaller in size compared to white adipocytes (Figure 1). Thus, the reduction in size of white adipocytes outlines the first step of their transdifferentiation into brown adipocytes without triggering apoptosis based on critical size [6]. Physiologically, adipocyte cell death is related to the growth up to the critical death size and the critical change in size for visceral adipocytes is smaller than that of subcutaneous adipocytes, which explains for the greater morbidity associated with visceral adiposity.The recent animal studies have outlined the plasticity of the adipose organ - the transformation into brown adipose tissue from white adipose tissue occurred in 
rats treated with ss3AR agonists is due to a direct transformation of differentiated unilocular adipocytes. The study endorsed the possibility to modulate the plasticity of the adipose organ for therapeutic interventions for obesity and related disorders.The so called browning phenomenon is associated withmodulation by several molecules and signalling pathways involved in the WAT physiology.

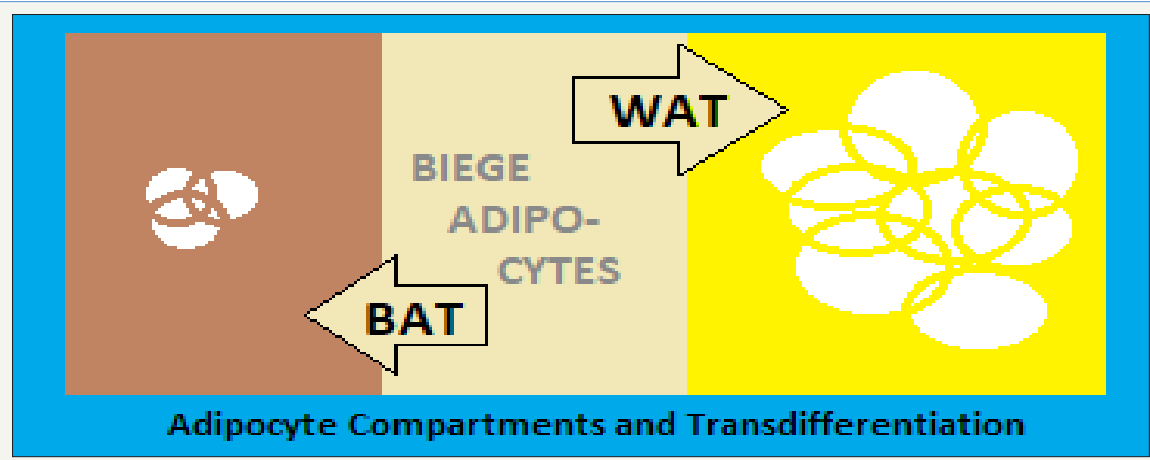

Figure 1: The adipose organ: composition and gross dynamics.

The adipose organ is adequately innervated, enabling it to respond tophysiological, environmental and homeostatic stimuli throughtheCNSinterface.The nerve supply reaches the vasculature and adipocytes, by noradrenergic fibers expressing tyrosine hydroxylase (TH). The thermogenesis is stimulated through sympathetic nervous system activationon exposure to a subnormal temperature andnor epinephrine acting on $\beta$-3-adrenoreceptors topromote and facilitate the molecular pathway for thermogenesis in BAT, where the density of TH- fibers is higher than in WAT. Further, ona chronic exposure to colder temperature, the density of the TH-fibers has been shown to increase simultaneously with the increase in brown adipocytes [7].In this context, the human BAT consists of UCP1-expressing adipocytes, densely innervated by THimmuno-reactive fibers [8].

Phylogenetically, the brown and white adipocytes are derived from adipose tissue-vascular endothelial cells [9]. Both of the two adipocytes are contained collectively in the fat depots, though their ratio is dynamically changing.The higher amounts of WAT are associated with obesity and related disorders, whereas a higher number of brown adipocytes is inversely related to adiposity, MetS, IR and related disorders.The plasticity is an integralquality of the adipose tissue and the trans-differentiation process is related to physiological conditions like chronic exposure to cold, physical exercise, pregnancy and lactation and pathological conditions like over-nutrition and obesity. A recently identified hormone, irisin, is produced by skeletal muscle following physical exercise. Irisin induces browning of WAT in mice [10].Irisin is also produced in humans and projected to be manipulated to modulate plasticity of the adipose organ in selected population group.

\section{Mechanisms of adipose plasticity}

The WAT expresses $\beta-3$ adrenoceptors ( $\beta 3 \mathrm{ARs}$ ), essential for the browning phenomenon, as proved by blunted browning in $\beta 3 A R$ knockout mice. In addition, other mechanisms are alsoassociatedwithtrans-differentiation of WAT to BAT and adipose tissue browning, like - Activation of peroxisome proliferatoractivated receptor- $\gamma$ (PPAR $\gamma$ ), Stimulated activity of fork-head box protein $\mathrm{C} 2$ (FOXC2) increasing the sensitivity of the $\beta$-adrenergic cAMP-PKA signaling pathway, Activation of cyclooxygenase 2 (COX2), Activation of the microRNA cluster comprising miR193b-365, Activation of the growth factor FGF21, Inhibition of retinoblastoma $(\mathrm{Rb})$ protein activity, and Induction of myokine hormone, irisin.The 'brown-like adipocytes' (BLAs) arise from the WAT following certain physiological stimuli. The BLAs have been projected to emerge from pharmacological therapy. The BLAs are UCP-1expressing adipocytes, a feature of the BAT. Experimentally, the lipopolysaccharide (LPS)-challenged macrophage conditioned medium and TNF- $\alpha$ inhibit theUCP-1 mRNA induction in adipocytes through extracellular signal-regulated kinase (ERK) activation and the blockade of nuclear signaling has been shown to suppress the emergence of BLAs from white adipocytes [11].

Epinephrine, actsthrough the $\beta$-adrenergic receptors, cAMP, protein kinase $A$ and nuclear signaling, to facilitate the expression of UCP-1 mRNA in adipocytes. The BLAs emerging from the WAT improve insulin sensitivity and IR, and boost and facilitate the norepinephrine-induced oxygen consumption to halt apoptosis and adipocyte survival. The physiological actions of BAT lead to suppression of weight gain, a healthy phenotype, and improved homeostasis of glucose and lipid metabolism. On the other hand, the lack of the BAT activity predisposes toadiposity and metabolic disorders [12]. Thus, the projected possibility to manipulate the plasticity of the adipose organ holds a therapeutic potential. It has already been shown that animals with higher BAT are comparatively resistant to adiposity and T2DM, whereas those with lower BAT ratio are prone to adiposity and T2DM [13]. Further, weight gain in rats as well as in human volunteers is restrained when they are treated with $\beta 3 \mathrm{AR}$ agonists, which are capable of inducing the browning in WAT [14].

The lactation represents a special phenomenon involving adipose organ plasticity. In pregnancy and later during lactation, the subcutaneous adipose depots in female mice convert into milk-secreting glands, a hormone-regulated process called adipo-epithelial trans-differentiation. There occurs a progressive 
reduction in the adipocytes and a simultaneous rise in the epithelial cells forming adenomeres of mammary glands. The process being reversible, the epithelial-adenomeres cells revert back to adipocytes in the post-lactation period [15].

\section{The endocrine role of adipose organ}

Apart from being storage depots for somatic energy, adipose organ has endocrine function, and plays an important role through the adipocytes-derived hormones in metabolic homeostasis. The adipose tissues secrete several peptides termed adipokines, acting in an autocrine, paracrine and endocrine modes to influence the metabolic functions. So far, over 50 adipokines have been discovered and profiled. Of these,adipokines, like adiponectin and leptin are of major significance. Both, adiponectin and leptin have wide-ranging central and peripheral effects on metabolism and energy homeostasis. Adiponectin and leptin, both, reduce circulating fatty acids and triglyceride level through increased fat oxidation. The latter effect is mediated by activating the enzyme AMP-activated protein kinase (AMPK), which increases glucose transport in muscle [16]. Interestingly, the exercise also activates AMPK, which can increase lipo-oxidation and reduces IR. The adipocyte hormones and exercise appear to act via a similar signal transduction pathway to increase lipo-oxidation, reduces IR and improve insulin sensitivity [17].

Adiponectin (adipoQ): Adiponectin (adipocyte complementrelated protein - adipoQ) is vastly expressed in adipocytes. It improves insulin sensitivity in liver and muscles, lowers IR, increases FFA oxidation in muscles and other tissues, and decreases serum glucose, FFA and triacylglycerol concentrations.In animal models,the experiments in insulin-resistant mice treated with adipoQ glucose tolerance and IR improve. In human volunteers, plasma adipoQ levels have been shown to fall with obesity, the effect being greater in men than in women [18]. The reduced adipoQ concentrations correlate with IR and hyperinsulinemia. In addition, several polymorphisms of the adipoQ gene (APM1, mapped to chromosome 3q27) have been identified which are associated with reduced plasma adipoQ activity and increased risk of T2DM, IR and MetS [19]. In addition, adipoQ influences the development of atherosclerosis and adipoQ concentration is shown to be reduced in patients with CVD. The adipoQ inhibits theTNF- $\alpha$ induced expression of adhesion molecules and the transformation of macrophages to foam cells, the key players in the components of atherogenesis process [20].

Leptin: Leptin influences the appetite and food intake through a direct effect on the hypothalamus, whereas the plasma leptin concentrations correlate with BMI [21]. The role of leptin in regulating body weight is, thus, complex. In an obese person, the increased amount of leptin-secreting adipose tissue lets the serum leptin levels are high [22]. With increased leptin concentrations, the target cells increasingly express SOCS-3 (suppressor-of-cytokinesignaling), a potent inhibitor of leptin signalingand become resistant to leptin action [23]. The feedback mechanism, thus, results in loss of effectivity of leptin in obese individuals despite its high circulating levels.

Experimentally, the mice lacking the gene coding for leptin (ob/ ob mice) who are obese and diabetic, on treatment with leptin, reduce food intake, increase metabolic rate and lose weight [24]. Similarly, the mice with a genetic mutation affecting the leptin receptor in the hypothalamus also exhibit comparable features [25]. A mouse model of congenital lipodystrophy, having IR, hyperinsulinemia, hyperglycemia and fatty liver, leptin therapy reversed the insulin resistance and diabetes can be mentioned in this context [26]. Another, rare disease, namely the lipoatrophic diabetes, has little or no fat mass, reduced serum leptin and elevated serum triglyceride. In a pilot study, administration of exogenous leptin to individuals with lipoatrophic diabetes has shownremarkable reductions in triglyceride level, liver volume and glycated hemoglobin [27].

\section{The Adiposity Metabolic Mechanisms}

\section{Overnutrition and nutrient overload}

The diet-gene interaction is an important determinant of health [28]. The MetS encompassing abdominal adiposity, IR, dyslipidaemia and hypertension, accounts for a major public health threat both indeveloped as well as developing regions of the world [29]. The rise in obesity and MetS prevalence in recent decades isattended by the background changes in dietary patterns, reflecting increased nutrient availability and consumption [30]. In general, diets rich in fat and rapidly-digestible carbohydrates have increased average calorie intakeand led to over-nutrition and nutrient overload culminating into global obesity epidemic (Figure 2).

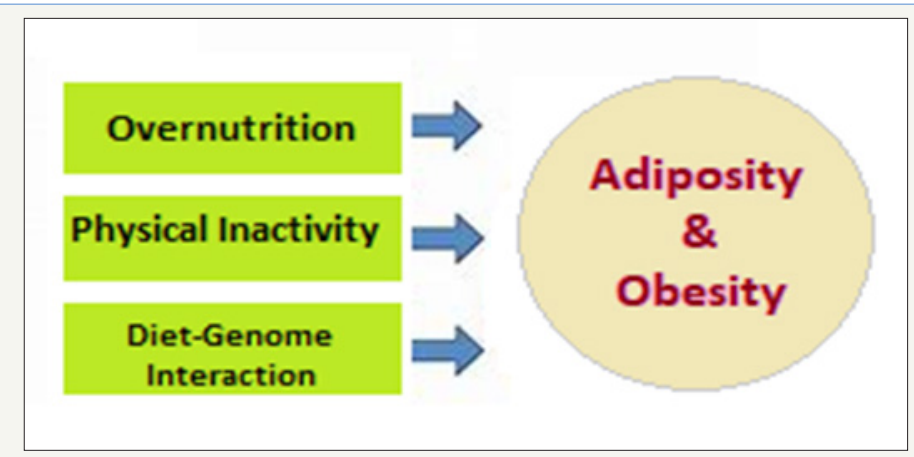

Figure 2: Over nutrition, nutrient overload and adiposity. 
The pooling of positive energy balance, that is,food and energy intake greater than energy expenditure, leads to expansion of the WAT, occurring throughboth an increase in adipocytes volume as well as the development of new WATtransdifferentiate from BAT to store additional energy[31]. Further, the energy accumulation inducesthe TGF- $\beta /$ Smad3 signaling pathway as compared to the experimental knockdown of Smad3 (a component of the TGF $\beta$ signaling pathway) in mice which induces browning of the adipose tissue and appears to protect from obesity and diabetes [32].

\section{The protective role of adipose tissue}

Physiologically, the role of adipose organ as depots for energy storage appears to be an evolutionary need. An obese animal is evolutionary suitable to survive by utilizing the fat depots in an adverse milieu when food is scarcely obtainable, as exemplified by in penguins. Similarly, in the evolutionary phases as hunter and food-gatherer, the fat depots stored nutrients for times when food availability became erratic. The adipose tissue remained in a dynamic equilibrium, overnutrition led to filling of adipose depots, conversion of BAT to WAT to store more nutrients, the WAT was converted to BAT to sustain during the cold weather, and the stored nutrients helped the animal survive in the phase of scarce- or nonavailability of food.

The stored nutrients and their metabolites, nutrients such as cholesterol, folic acid, vitamin E and vitamin D, and ligands for various transcription factors are expressed in adipose tissue. The fatty acids ingested by adipocytes interact with numerous transcription factors critical for various developmental and metabolic processes like, PPAR- $\alpha,-\gamma$ and -delta, sterol regulatory element-binding proteins 1 and 2 and liver $X$ receptors $\alpha$ and $\beta$. The glucose is also stored as fatty acids via lipogenesis in adipocytes. The metabolic signals from the adipose organ are released as peptides called adipokines, which play an important role in metabolic regulation.

The physiological protective role:Rather than total body fat mass (BFM), the body fat distribution (BFD) including visceral, subcutaneous and ectopic fat, and the impaired adipocyte function are predictable of IR, T2DM and related complications at the individual level [33]. In humans, the abdominal obesity has been linked with morbidity and is a predictor of the mortality risk Further, the fat deposition in ectopic zones like liver and skeletal muscle causes lipotoxicity and impairment to insulin action. On the other hand, the expansion of SF may confer a relative protection from metabolic derangements [34].

In general, the BFM and BMI are important factors which determine the metabolic healthand the BFD and adipose tissue dysfunction are major determinants for genesis of adiposity-related IR and cardio-metabolic disease. A subgroup of obese persons, called 'metabolically healthy obese' (MHO), appears to have a less ectopic fat storage, a better adipose tissue function or a less marked adipose tissue dysfunction and a less pronounced IR than metabolically unhealthy obese individuals. This observation emphasizes the important role of adipose tissue function in metabolic health. As such, the MHO individuals have a less pronounced central obesity and a more subcutaneous fat distribution, though on BMI scale they may have similarity with metabolically unhealthy obese person. The MHO individuals are relatively protected against chronic metabolic disorders compared to metabolically unhealthy obese individuals. Still, the MHO persons have an increased risk of developing obesityrelated disorders and complications as compared to normal weight individuals. Adipose tissue also contains immune cells, both the adaptive (B and T lymphocytes) and innate (macrophages), making it an immune organ.

The pathophysiological protective role:The over-nutrition leads to nutritional overload, which later, in due course causes nutritional toxicity endangering the intracellular organelle and intracellular as well as intercellular metabolic processes. This avoided by a selective and differential development of IR by various tissues to limit the excess nutrient entry into their cells. The plasticity of the adipose organ by modifying its cells come to aid and protects various vital organs. The WAT helps in dealing with the nutritional overload by enlarging their cells to store more as well as by trans-differentiation to produce more WAT from BAT. The adipose tissue, thus, plays an important role to protect several vital organs from the challenge posed by nutritional overload, and manifest as adiposity. The positive energy balance entails the calorie intake higher than actual energy expenditure, leads to expansion of the WAT, occurring through an increase in the volume as well as numbers. The adiposity can, thus, be regarded a temporary way to deal with nutritional excess.

Storing of excess energy, in due course, leads to overweight and obesity, and associated disorders, such as IR, which are linked with abnormal adipose tissue amount and its altered physiology to which several factors contribute [35]. The metabolic function of adipocytes changes as they enlarge with increasing obesity. First, adipocytes function to maintain temperature through thermogenesis and acts asstorehousesof nutrients including free fatty acids (FFAs). Second, they release adipokines to regulate BFM and modulate appetite and food intake. Third, with increasing adiposity, as they enlarge the regulating mechanisms become dysregulated, the in the adipose tissue, and inflammation ensues. Finally, with the enlargement of individual adipocytes and the adipose tissue mass, the tissue oxygenation is hampered culminating as areas of inflammation and apoptosis and the enlarged, disarrayed and damaged adipocytes attract macrophages and promote inflammation, culminating in release of proinflammatory markers that predispose to IR and other metabolic dysfunctions [36].

\section{The inflammatory effects of adiposity}

Adipocytes cellular and molecular alterations:The impaired adipose tissue expansion, adipocyte hypertrophy, altered lipid metabolism and local inflammation lead to adipose tissue dysfunction. The recent studies suggest that impaired adipose tissue oxygenation may be a key factor. With obesity, apart from the adipocytes enlargement, and adipose tissue endures molecular and cellular alterations which affect the global metabolism. The fasting FFAsare increased due to a deficiency of perilipins, the phosphoproteins present on the surface of triacylglycerol droplets acting as 
gatekeepers for release of FFAs. The excess FFAs release from in the enlarged adipocyte promotes IR in tissues like muscle [37].The macrophage infiltration occurs mainly in the visceral adipose tissue following impaired adipose tissue oxygenation, injury to adipocytes and their apoptosis, leading to inflammation and release of various cytokines (Figure 3), which contribute to IR and related disorders associated with obesity [38].The macrophages form crown-like structures (CLSs) to surround the dead adipocytes, which are then phagocytosed by the macrophages [39].Macrophage infiltration has also been correlated with the size of adipocytes in visceral fat depots. Visceral fat adipocytes are smaller than subcutaneous ones but have a smaller critical death size (CDS), hence die at a smaller CDS [40]. This observation explains the deleterious metabolic fallouts of excess visceral fat accumulation.

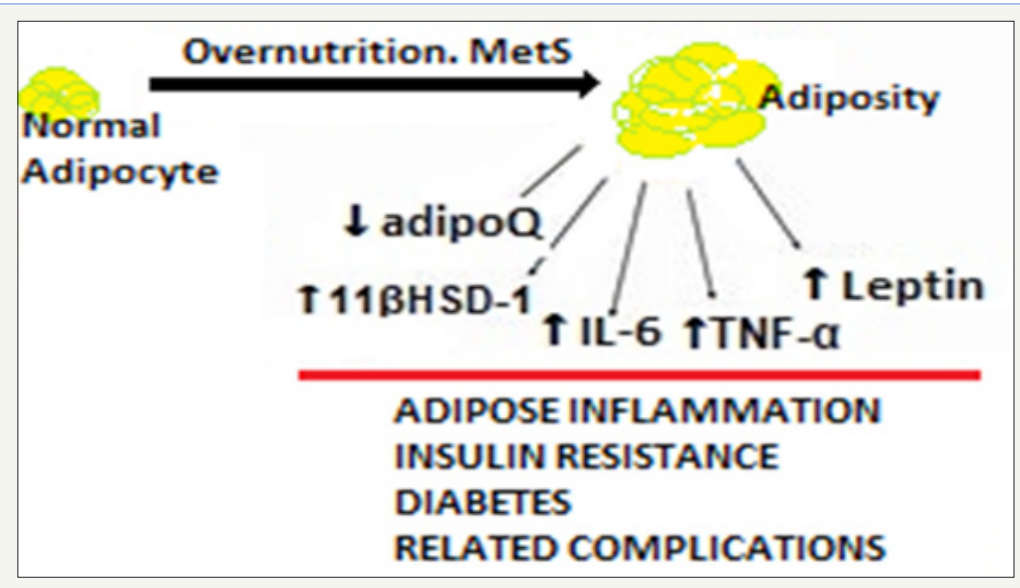

Figure 3: Adiposity, adipotoxicity and adipo-inflammation.

Adiposity and proinflammatory factors: Following adipocyte apoptosis and adipose tissue inflammation, the macrophages increasingly accumulate in the visceral adipose tissue [41] and are responsible for increased cytokine production and release [42].

The proinflammatory factors and IR: With adiposity, variousproinflammatory molecules, factors and co-factors are increasingly produced by adipose tissue including TNF- $\alpha$, IL6 ,IL-1 $\alpha$,monocyte chemotactic protein 1 , inducible nitric oxide synthase, transforming growth factor $\beta 1$, pro-coagulant proteins such as plasminogen activator inhibitor type 1, tissue factor, factor VII and C-reactive protein [43]. The appearance of the inflammatory molecules and their propensity to promote IR and other complications of obesity like T2DM are closely linked [44]. The macrophage-released pro-inflammatory cytokines interfere with the insulin signaling [45].

On basis of recent research, the sequence of events linking obesity with IR, apparently, can be outlined as follows:Adipocyte hypertrophy due to obesity $\rightarrow$ Adipocyte stress and metabolic dysregulation possibly involving hypoxia [46] $\rightarrow$ Production of chemo-attractants $\rightarrow$ Macrophage infiltration of adipose tissue $\rightarrow$ Thedeath of adipocytes on reaching the CDS, predisposed by decreased oxygenation $\rightarrow$ Reabsorption of adipocyte remnants by macrophages $\rightarrow$ Explosiverelease of cytokines by macrophages $\rightarrow$ Increased level of circulating cytokines $\rightarrow$ IR in peripheral tissues.

The TNF- $\alpha$, IL-6 and 11ßHSD-1: The TNF- $\alpha$ concentrations in adipose tissue have been correlated with obesity and IR in patients with and without T2DM [47].Also,TNF- $\alpha$ messenger RNA expression in adipose tissue has been correlated with fasting plasma glucose, insulin levels and FFA concentrations. The TNF- $\alpha$ increases adipocyte lipolysis and may increaseIR by promoting the release of fatty acids from adipose tissue into the bloodstream to act on tissues such as muscle and liver. Thus, adipose tissue TNF- $\alpha$ which acts locally in adipose tissue, in due course promotes IR in muscles and other peripheral tissues as well.The IL-6 expression isincreasedandhigher in visceral than in peripheral adipocytes. IL-6 increases lipolysis and fat oxidation and causes IR in the liver [48], and its elevated plasma concentration correlate with IR [47], which is a predictor for development of T2DM and for CVD [49].

The concentration and activity of 11ß-hydroxysteroid dehydrogenase type 1 (11ßHSD-1) are elevated in adipose tissue of obese individuals [50].The11ßHSD-1 converts inactive metabolites of cortisol back into cortisol,resulting in increased local adipose tissue concentrations despite normal plasma cortisol levels. In the animal model experiments, over-expression of $11 \beta \mathrm{HSD}-1$ in mice resulted in increased visceral adiposity and metabolic abnormalities likeIR and diabetes, increased cytokine expression, hyperphagia, hyperlipidaemia, and hypertension [51]. These complications observed mice are similar to those in obese humans. There arenumerous inflammatory factors associated with the development of obesity related complications and development of the chronic diseases associated with obesity.

\section{The excess folic acid and obesity link}

The standardrecommended daily requirement of folic acid is $400 \mu \mathrm{g} /$ day for the general adult population. Sources of natural folates (pteroyl-polyglutamates) include green leafy vegetables, legumes and oranges.Simultaneously, there is provision of fortification of staple foods. The widespread supplement use has increased folic acid intake in urban diets. Children and elderly populations are likely to have high folic acid intake because large proportions of their diet consist of cereals and bread.The pregnant 
women and lactating mothers may have high intakes due to high supplement use.The excess folic acid intake raises concerns about possible adverse effects, including alterations in energy and lipid metabolism as documented in certain recent studies.The folic acid appears to influence energy and lipid metabolism by modulating DNA methylation and gene expression patterns [52]. There is evidence that influence of methyl donors, including folic acid, on gene expression may be tissue-, site-, and gene-specific. The gene expression analysis has shown increased mRNA levels of PPAR $\gamma$ and some of related target genes.

The excess folic acid intake may promote changes to one carbon metabolic pathways and gene expression patterns, leading to liver injury. In an animal model, the effects of excess folic acid (EFA) with a high fat (HF) diet were related to significantly higher weight gain andincrease in fat massas compared to rats fed adequate folic acid (AFA). There was also present increased inflammation along with impaired glucose tolerance in HF-EFA fed rats, as compared to high fat-adequate folic acid (HF-AFA) fed rats. The results, thus, suggest that EFA may exacerbate weight gain (Figure4), fat accumulation, and inflammation caused by consumption of a HF diet,simultaneously [53]. The observational evidence has also linked folate status to adiposity and altered lipid and energy metabolism [54]. Obesity and the metabolic syndrome represent a major public health burden and the link between EFA and metabolic complications warrants further investigations [55].In addition, the observational evidence has linked high dietary folic acid intake to increased risk of colorectal and prostate cancers, impaired immune function and cognitive decline.

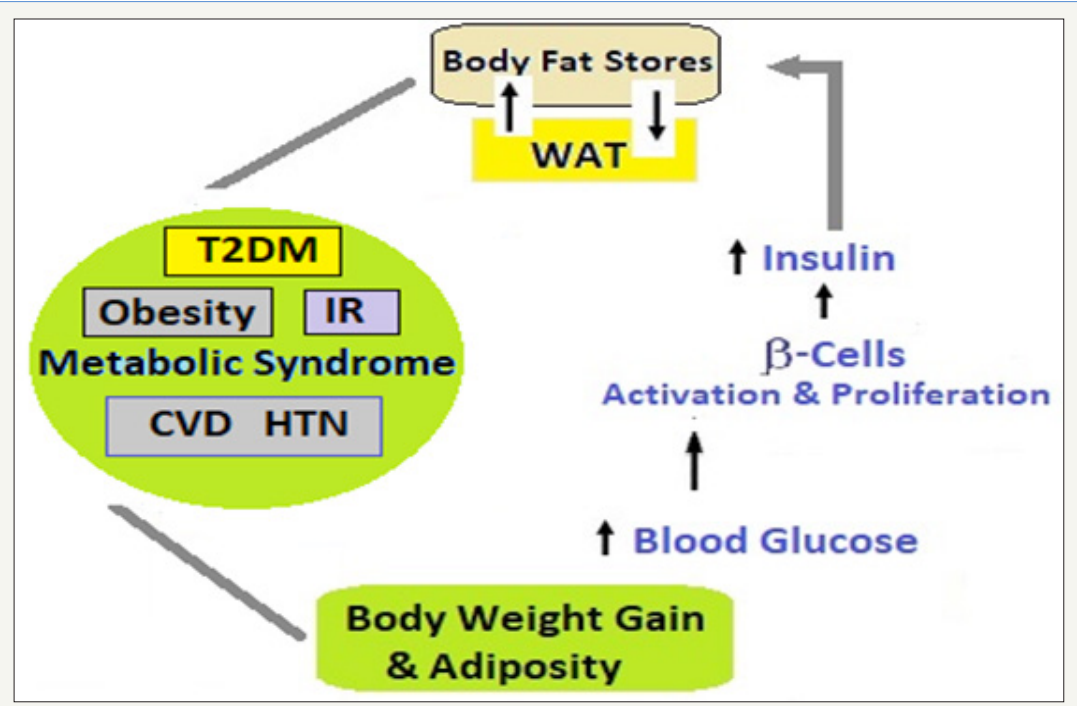

Figure 4: Metabolic changes triggered by adiposity.

\section{Adiposity, IR, T2DM and Other Fallouts}

\section{The global epidemic of adiposity}

There is an alarming increasedprevalenceofoverweight and obesity in developed and developing regions the world-over [56]. The obesity being an important risk factor for DM, CVD, AD, cancer and premature death, its high prevalence makes it a global health challenge. In general, the prevalence of obesity tends to increase with age, peaks during middle-age and decreases thereafter, simultaneously, its consistently higher among women compared with men in similar age groups and the projections for thehealth burden and the future trends indicate that the numbers of obese people will increase to about $44-45 \%$ by year 2030 [57]. Further, compared with developed regions of the world, developing regions are being projected to have a higher proportional increase in the number of obese individuals by 2030. Growth in population size, population aging, urbanization and changes in lifestyle encompassing increased calorie intake and physical inactivity, all seem to contribute to an impending epidemic of obesity in the developing regions [58].

\section{The metabolic fallouts of adiposity}

The BMI and adiposity have been correlated with obesityrelated complications. The adipose organ accounts for about $20 \%$ of total body mass in lean individuals and for over $50 \%$ in those having morbid obesity.The adipose organ is a biologically active tissue which secretes various adipokines and stores nutrients such as free fatty acids [59]. The adipose tissue organ is constituted of white, beige and brown adipocytes serving specialized functions, and their coordinated actions ensure an optimal metabolic homeostasis. The complications ensue when the coordinated action of the three adipocytes groups becomes dysfunctional as a result of excess nutrients load, IR and associated metabolic dysfunctions leading to lipo-toxicity and compromised global metabolic homeostasis.

The risk stratification at baseline can improve the effectiveness of a certain interventions in a particular subgroup of the population. This has been endorsed by an analysis of data from the TULIP study, demonstrating that stratification of subjects with prediabetes by phenotype (including impaired insulin secretion, IR and nonalcoholic fatty liver) at baseline predicts the effectiveness of a lifestyle intervention in achievingthe therapeutic goals [60]. The 
study also demonstrated that a high-risk phenotype at baseline was associated with reduced improvement in glycemia following lifestyle intervention.

\section{The adiposity and T2DM}

The adiposity and T2DM link:The adiposity is a majorrisk factor for development of metabolic disorders including diabetes and atherosclerosis. Further, obesity has been linked to various medical, psychological and social conditions, the most devastating of which is T2DM.Obesity and T2DM, both, are associated with IR. However, not all obese persons, though having IR to varying degree, do not develop diabetes, because at thatstage the $\beta$-cells are able produce adequate insulin to override and overcome IR, as one of the metabolic changes triggered by adiposity.

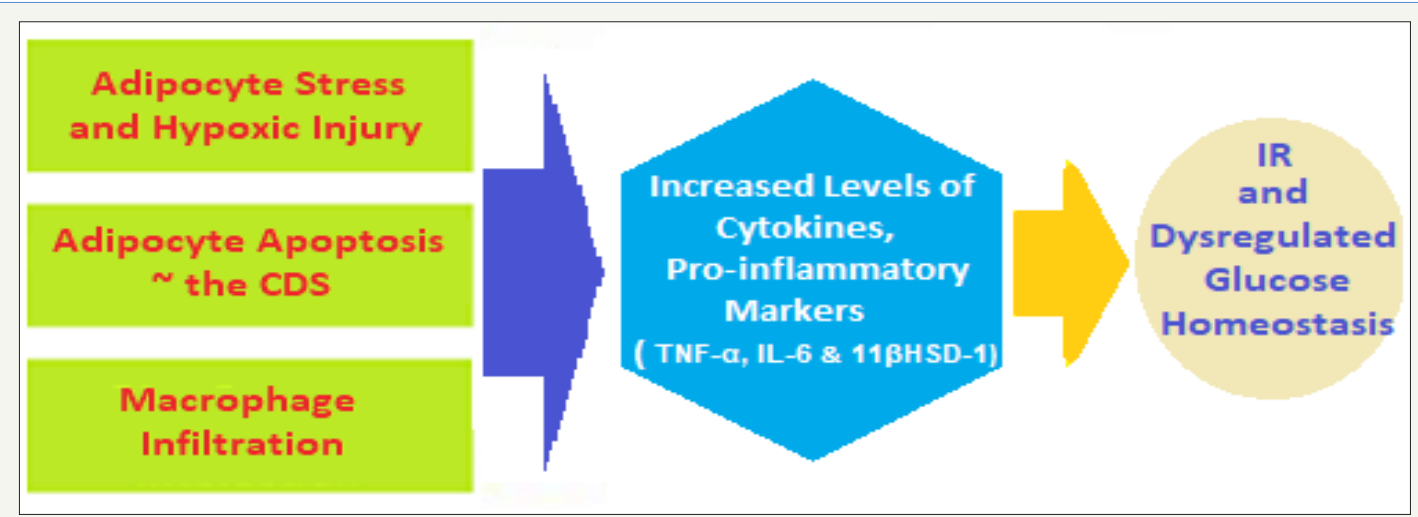

Figure 5a: The obesity link with IR-Adiposity/Obesity $\rightarrow$ Adipocyte stress and hypoxia $\rightarrow$ Production of chemo-attractants $\rightarrow$ Chemoattraction and infiltration of macrophages $\rightarrow$ The adipocyte apoptosis on reaching the CDS $\rightarrow$ Reabsorption of adipocyte remnants by macrophages $\rightarrow$ Massive release of cytokines by macrophages $\rightarrow$ Increased circulating cytokines like TNF-a, IL- 6 and $11 \beta$ HSD- $1 \rightarrow$ IR in peripheral tissues $\rightarrow$ Initiation of T2DM.

The main offending factor to impair the insulin sensitivity leading to IR is held the increased release of NEFAs seen both in T2DM and in obesity (Figure 5a). Conversely, with decreasedserum NEFA levels, as in the case with antilipolytic drugs, the peripheral insulin sensitivity, glucose uptake and utilizationare improved [61]. The insulin sensitivity is also influenced by another crucial factor, the BFD. Thus, even a lean individual with adverse BFD, that is, having increased abdominal or central fat despite BMI in a normal range, may have IR and its fallouts. Individuals with more peripheral BFD than central retain better insulin sensitivity than individuals with adverse central BFD [62].

The researchers, Marcial et al.[64] from their study in Puerto Ricans and other Hispanic populations have explained the subcellular and molecular mechanisms of IR, adipose tissue inflammation and the development of DM [63].As a hormone, insulin has anabolic effects and enhances glycogen synthesis in liver and muscle as well as enhances protein synthesis and inhibits proteolysis. Thus, under influence of insulin, IR is indirectly altered by fat storage and mobilization or any change in BFD [64].

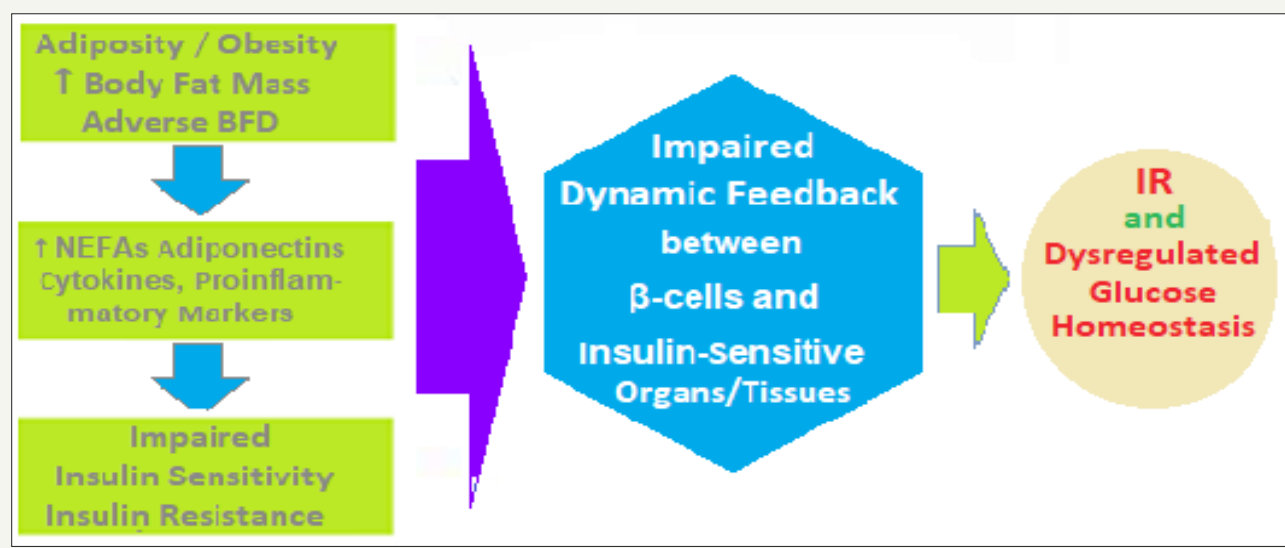

Figure 5b: Adiposity, Obesity, $\uparrow$ Body Fat Mass and BFD- $\uparrow$ Levels of NEFAs, glycerol, adiponectins, cytokines, proinflammatory markers like NNF- $a$ and IL- 6 and $11 \beta$ HSD- $1 \rightarrow$ Impaired insulin sensitivity (influenced by BFD)+IR. The Impaired dynamic intricate feedback relationship between the $\beta$-cells and the insulin-sensitive tissues $\rightarrow$ dysregulation of glucose levels $\rightarrow$ Development of T2DM. Continued decline in $\beta$-cell mass and function leading to inadequate insulin secretion worsens PP and F blood glucose levels and DM.

\section{The $\beta$-cells, insulin sensitivity and IR}

In normal conditions, there is a dynamic feedback relationship between the $\beta$-cells function and the insulin-sensitive tissues. For the stability of glucose levels, any changes in insulin sensitivity iscoordinated by anequivalent change in circulating insulin levels. Any failure of this intricate process results in a dysregulation of 
glucose levels and the development of DM.Thecontinued decline in $\beta$-cell function is the main cause of development and worsening of T2DM (Figure 5b). When there is inadequate insulin secretion due to $\beta$-cell dysfunction, first, postprandial blood glucose and later fasting blood glucose levels are affected. In due course, there is decreased hepatic and muscle glucose uptake and impaired inhibition of liver glucose production. The high blood glucose levels lead to glucotoxic effects on the $\beta$-cells and worsens IR and insulin sensitivity [65]. The occurrence of IR and a failure of the compensatory secretory mechanism by $\beta$-cells in humans contributes to increased amount of NEFA levels produced lipids by adipocytes. Through a vicious loop, the increased plasma NEFA levels cause a further loss of already compromised $\beta$-cells function.

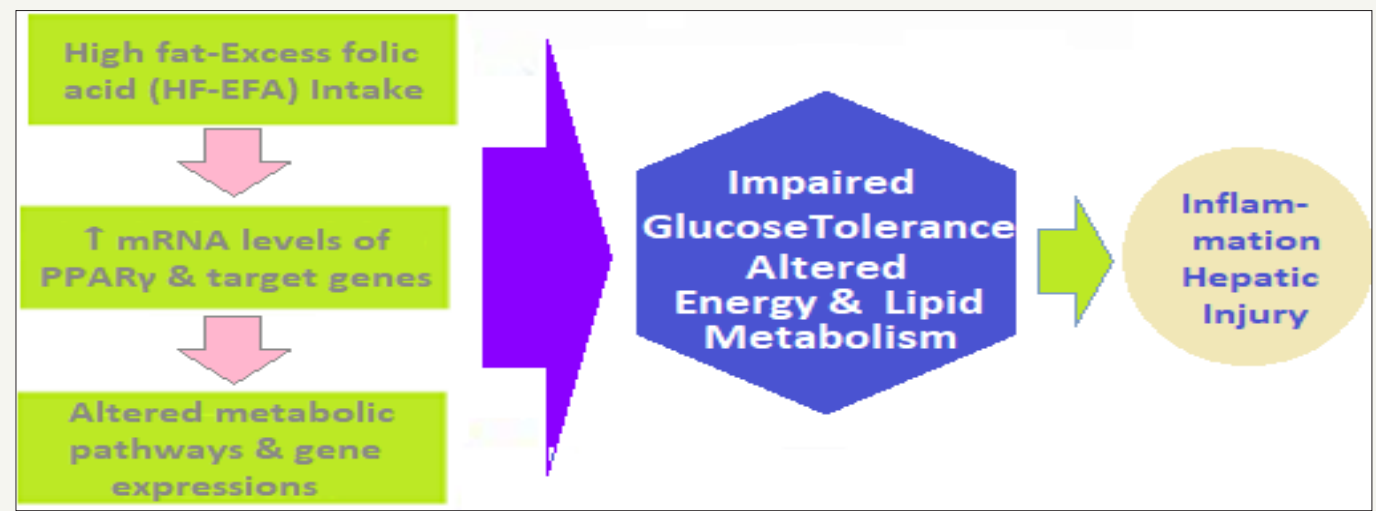

Figure 5c: High Dietary Fat and Excess Folic Acid Intake - The high fat-excess folic acid intake (HF-EFA) $\rightarrow$ excess folic acid Levels $\rightarrow$ increased mRNA levels of peroxisome proliferator-activated receptor $\gamma$ (PPARY) and some of its target genes $\rightarrow$ greater weight and fat mass gain $\rightarrow$ increased inflammation along with impaired glucose tolerance + alterations in energy and lipid metabolism in adulthood $\rightarrow$ Altered carbon metabolic pathways and gene expression patterns $\rightarrow$ Fatty Liver injury, Weight gain and obesity, and IR and Diabetes mellitus.

(Figure 5c) - High dietary fat and excess folic acid intake - The high fat-excess folic acid intake (HF-EFA) $\rightarrow$ excess folic acid Levels $\rightarrow$ increased mRNA levels of peroxisome proliferatoractivated receptor $\gamma$ (PPAR $\gamma$ ) and some of its target genes $\rightarrow$ greater weight and fat mass gain $\rightarrow$ increased inflammation along with impaired glucose tolerance+alterations in energy and lipid metabolism in adulthood $\rightarrow$ Altered carbon metabolic pathways and gene expression patterns $\rightarrow$ Fatty Liver injury, Weight gain and obesity, and IR and Diabetes mellitus.

The high BMI, T2DM and IR are closely linked. With obesity, the levels of NEFAs, glycerol, adiponectins, cytokines, proinflammatory markers like NNF- $\alpha$ and IL-6, and certain other substances involved in the genesis of IR, are increased [66]. The pathogenesis of development of T2DM involves the impairment of $\beta$-islet cells heralding a lack of blood glucose control. The development of T2DM is sets-in when the $\beta$-islet cells failure is accompanied by IR [67].Adiposity and increased BFM contribute altered T2DM.

\section{Adiposity, T2DM and obesity factors}

The rising incidence of T2DM entails role of certain hitherto novel factors like, the increased prevalence of obesity among all age groups and physical inactivity, altered dietary habits and urbanization [68]. IR with lipid and thrombotic abnormalities and atherosclerotic risk factors like, smoking, family history and hypertension, determine the $\mathrm{CV}$ risk, which is associated with the development of IR even before apparent hyperglycemia develops. The hyperglycemia contributes to microvascular and metabolic complications, but the macrovascular complications are not directly linked to glycemic abnormalities. An important concept, the 'ticking clock' hypothesis by Haffner et al.[69]outlined that the onset of hyperglycemia puts one at risk for developing diabetic macrovascular complications at an earlier point, at the genesis of IR and well-before apparent hyperglycemia [69].Another issue, putforth by Wilkin et al as the 'accelerator hypothesis' is an accepted theory linking the association between body mass and DM [70].The increasing body weight accelerates the risk of developing IR and DM in persons predisposed genetically to DM. There has beennoted an inverse relationship between BMI and the diagnosis of DM. With body weight gain, DM appears to be diagnosed earlier, a fact applicable to both T1DM as well as T2DM.

\section{Conclusion: Future Projections and Options}

\section{Adiposity/obesity and T2DM: Projections for the future}

Both obesity and T2DM represent a global health crisis brought about by a rampant urbanization, the nutrition transition from traditional to modern and an increasingly sedentary lifestyle. Preventing, controlling and treating Obesity as well as T2DM and their fallouts require a thoughtful long-term planning and rational allocation of resources, especially in countries with limited resources. The accumulating evidence over several decades, stronglyputs-forth that the majority of T2DM and obesity both can be prevented through dietary and lifestyle modification. In general, the public health strategies to target the obesogenic environment are critically needed.Translating the clinical and epidemiologic findings into practice will require fundamental shifts in public policies and health systems.To expedite improvement in therapeutics to obesity and T2DM at individual level, there is need for the innovative approaches to their pharmacological and nonpharmacological management includingthe bariatric surgery. 


\section{Adiposity/obesity and T2DM: The therapeutic options}

The therapeutic options for weight reduction: As the prevalence of obesity and T2DM continue to increase, the need for implementation of effective weight-loss strategies becomes more urgent. The lifestyle interventions have been shown to reduce body weight and improve risk factors related to obesity and T2DM. The results from various studies suggest that lifestyle modifications, may represent a safe and effective therapy for the management of obesity in patients with T2DM.The excess body weight is an established risk factor for T2DM, yet most obese individuals do not develop T2DM. Recent studies continue to identify the links between obesity and T2DM involving proinflammatory cytokines (TNF- $\alpha$ and interleukin-6), insulin resistance, deranged fatty acid metabolism, and cellular processes such as mitochondrial dysfunction and endoplasmic reticulum stress even a modest weight reductionwhether through lifestyle/behavioral interventions, obesity medications, or bariatric surgerycan improve glycemic control and reduce risk related to T2DM and its complications.

Projecting therapeutic options for T2DM and weight reduction: The rising prevalence of obesity and T2DM has led to robust commercial interests in the development of therapeutic interventions for T2DM and weight reduction. As far as the pharmacotherapy is concerned, owing to the range of therapeutic options available, the treatment algorithm is complex. It appears that:

A. Metformin monotherapy will remain the first-line pharmacotherapy for T2DM. Metformin also activates AMPK, although metformin does not increase adiponectin concentrations like the TZDs. But, as relate to the drug tolerance issues, we need to develop a drug having advantages shown by metformin but bereft of its adverse effects including gastrointestinal intolerance.

B. The class of antidiabetic therapy called the thiazolidinediones (TZDs), for example, rosiglitazone, activate peroxisome proliferator-activated receptor $\gamma$ (PPAR- $\gamma$ ), a nuclear transcription factor, which is highly expressed in adipose tissue, reduces plasma FFA and glucose concentrations and improves insulin sensitivity. TZDs have also been shown to decrease $11 \beta$ HSD-1, increase adiponectin, decrease IL-6 and TNF- $\alpha$, and increase perilipin concentrations. The TZDs, similar to exercise, leptin, and adiponectin, have been shown to activate AMPK.

C. The emergence of several new antidiabetic drug-classes over the past decade, namely the Glucagon-Like Peptide-1 (GLP-1) receptor agonists, Dipeptidyl Peptidase 4 (DPP-4) inhibitors, and Sodium-Glucose Cotransporter 2 (SGLT-2) inhibitors, has led to considerable market growth. This is likely to affect considerably the use of previously available drugs like sulfonylureas and thiazolidinediones.

D. The insulin therapy is undergoing overhaul and a new and more patient-friendly approach is being evolved including therapeutic effects mimicking normal glycemic curve in vivo.

\section{References}

1. Haugen F, Drevon CA (2007) The interplay between nutrients and the adipose tissue. Proc Nutr Soc 66(2): 171-182.

2. Cinti S (2012) The adipose organ at a glance. Dis Model Mech 5(5): 588594.

3. Cinti S (2012) The adipose organ. Prostaglandins Leukot Essent Fatty Acids 73(1): 9-15

4. Park A, Kim WK, Bae KH (2014) Distinction of white, beige and brown adipocytes derived from mesenchymal stem cells. World J Stem Cells 6(1): 33-42

5. Cinti S (2011) Between brown and white: Novel aspects of adipocyte differentiation. Ann Med 43(2): 104-115.

6. Smorlesi A, Frontini A, Giordano A, Cinti S (2012) The adipose organ: white-brown adipocyte plasticity and metabolic inflammation. Obes Rev Suppl 2: 83-96.

7. Vitali A, Murano I, Zingaretti M C (2012) The adipose organ of obesityprone C57BL/6J mice is composed of mixed white and brown adipocytes. J Lipid Res 53(4): 619-629.

8. Zingaretti MC, Crosta F, Vitali A, Zingaretti MC, Frontini A, et al. (2009) The presence of UCP1 demonstrates that metabolically active adipose tissue in the neck of adult humans truly represents brown adipose tissue. FASEB J 23(9): 3113-3120.

9. Tran KV, Gealekman O, Frontini A, Zingaretti MC, Morroni M, et al. (2012) The vascular endothelium of the adipose tissue gives rise to both white and brown fat cells. Cell Metab 15(2): 222-229.

10. Boström P, Wu J, Jedrychowski MP, Korde A, Ye L, et al. (2012) A PGC1- $\alpha$ dependent myokine that drives brown-fat-like development of white fat and thermogenesis. Nature 481(7382): 463-468.

11. Sakamoto T, Takahashi N, Sawaragi Y, Naknukool S, Yu R, et al. (2013) Inflammation induced by RAW macrophages suppresses the UCP1 mRNA induction via ERK activation in 10T1/2 adipocytes. Am J Phsiol Cell Physiol 304(8): C729-C738.

12. Parinandi NL, Magalang UJ (2013) Avatars of Adipose Tissue-The saga of transformation of white fat, the villain into brown fat, the protector. focus on -inflammation induced by raw macrophages suppresses the Ucp1 MRNA induction via erk activation in 10T1/2 adipocytes. AJP Cell Physiology 304(8): C715-716.

13. Feldmann H M, Golozoubova V, Cannon B, Nedergaard J (2009) UCP1 ablation induces obesity and abolishes diet-induced thermogenesis in mice exempt from thermal stress by living at thermoneutrality. Cell Metab 9(2): 203-209.

14. Cypess AM, Kahn CR (2010) Brown fat as a therapy for obesity and diabetes. Curr Opin Endocrinol Diabetes Obes 17: 143-149.

15. Morroni M, Giordano A, Zingaretti MC, Boiani R, De Matteis R, et al (2004) Reversible trans-differentiation of secretory epithelial cells into adipocytes in the mammary gland. Proc Natl Acad Sci 101(48): 1680116806.

16. Yamauchi T, Kamon J, Minokoshi Y, Ito Y, Waki H, et al. (2002) Adiponectin stimulates glucose utilization and fatty-acid oxidation by activating AMP-activated protein kinase. Nat Med 8(11): 1288-1295

17. Ruderman N, Prentki M (2004) AMP kinase and malonyl-CoA: targets for therapy of the metabolic syndrome. Nat Rev Drug Discov 3: 340-351.

18. Arita Y, Kihara S, Ouchi N, Takahashi M, Maeda K, et al. (1999) Paradoxical decrease of an adipose-specific protein, adiponectin, in obesity. Biochem Biophys Res Commun 257(1): 79-83.

19. Kondo H, Shimomura I, Matsukawa Y, Kumada M, Takahashi M, et al (2002) Association of adiponectin mutation with type 2 Diabetes: A candidate gene for the insulin resistance syndrome. Diabetes 51(7): 2325-2328. 
20. Ouchi N, Kihara S, Arita Y, Maeda K, Kuriyama H, et al. (1999) Novel modulator for endothelial adhesion molecules: adipocyte-derived plasma protein adiponectin. Circulation 100(25): 2473-2476.

21. Farooqi IS, Keogh JM, Kamath S, Jones S, Gibson WT, et al. (2001) Partial leptin deficiency and human adiposity. Nature 414(6859): 34-35.

22. Considine RV, Sinha MK, Heiman ML, Kriauciunas A, Stephens TW, et al (1996) Serum immuno reactive-leptin concentrations in normal-weight and obese humans. N Engl J Med 334(5): 292-295.

23. Munzberg H, Flier JS, Bjorbaek C (2004) Region-specific leptin resistance within the hypothalamus of diet-induced obese mice. Endocrinology 145(11): 4880-4889.

24. Pelleymounter MA, Cullen MJ, Baker MB, Hecht R, Winters D, et al. (1995) Effects of the obese gene product on body weight regulation in ob/ob mice. Science 269(5223): 540-543.

25. Chua SC, Chung WK, Wu-Peng XS, Zhang Y, Liu SM, et al. (1996) Phenotypes of mouse diabetes and rat fatty due to mutations in the $\mathrm{OB}$ (leptin) receptor. Science 271(5251): 994-996.

26. Shimomura I, Hammer RE, Ikemoto S, Michael SB, Joseph LG, et al. (1999) Leptin reverses insulin resistance and diabetes mellitus in mice with congenital lipodystrophy. Nature 401: 73-76.

27. Oral EA, Simha V, Ruiz E, Andewelt A, Premkumar A, et al. (2002) Leptinreplacement therapy for lipodystrophy. N Engl J Med 346(8): 570-578.

28. McKay JA, Mathers JC (2011) Diet induced epigenetic changes and their implications for health. Acta Physiol (Oxf) 202(2): 103-118.

29. James PT, Rigby N, Leach R (2004) The obesity epidemic, metabolic syndrome and future prevention strategies. Eur J Cardiovasc Prev Rehabil 11(1): 3-8.

30. Cordain L, Eaton SB, Sebastian A, Mann N, Lindeberg S, et al. (2005) Origins and evolution of the western diet: Health implications for the $21^{\text {st }}$ century. Am J Clin Nutr 81(2): 341-354.

31. Hausman DB, DiGirolamo M, Bartness TJ, Hausman GJ, Martin RJ (2001) The biology of white adipocyte proliferation. Obes Rev 2(4): 239-254.

32. Yadav H, Quijano C, Kamaraju AK, Gavrilova O, Malek R, et al. (2011) Protection from obesity and diabetes by blockade of TGF- $\beta / S m a d 3$ signaling. Cell Metab 14(1): 67-79.

33. Gijs HG (2017) The metabolic phenotype in obesity: Fat mass, body fat distribution, and adipose tissue function. Obes Facts 10(3): 207-215.

34. Stefania CS, Pellegrinelli V, Vidal-Puig A (2017) Adipose tissue function and expandability as determinants of lipotoxicity and the metabolic syndrome. Obesity and Lipotoxicity 960: 161-196.

35. Strissel KJ, Stancheva Z, Miyoshi H, Perfield JW, DeFuria J, et al. (2007) Adipocyte death, adipose tissue remodeling, and obesity complications. Diabetes 56(12): 2910-2918.

36. Greenberg AS, Obin MS (2006) Obesity and the role of adipose tissue in inflammation and metabolism. The American Journal of Clinical Nutrition 83(2): 461-465.

37. Shulman GI (2000) Cellular mechanisms of insulin resistance. J Clin Invest 106: 171-176.

38. Weisberg SP, Mc Cann D, Desai M, Rosenbaum M, Leibel RL, et al. (2003) Obesity is associated with macrophage accumulation in adipose tissue. Clin Invest 112(12): 1796-1808.

39. Murano I, Barbatelli G, Parisani V, Latini C, Muzzonigro G, et al. (2008) Dead adipocytes, detected as crown-like structures, are prevalent in visceral fat depots of genetically obese mice. J Lipid Res 49(7): 15621568.

40. Virtue S, Vidal-Puig A (2010) Adipose tissue expandability, lipotoxicity and the Metabolic Syndrome - an allostatic perspective. Biochim Biophys Acta 1801(3): 338-349.

41. Weisberg SP, Mc Cann D, Desai M, Rosenbaum M, Leibel RL, et al (2003)
Obesity is associated with macrophage accumulation in adipose tissue. J Clin Invest 112(12): 1796-1808.

42. Xu H, Barnes GT, Yang Q, Tan G, Yang D, et al. (2003) Chronic inflammation in fat plays a crucial role in the development of obesity-related insulin resistance. J Clin Invest 112(12): 1821-1830.

43. Visser M, Bouter LM, Mc Quillan GM, Wener MH, Harris TB, et al. (1999) Elevated C-reactive protein levels in overweight and obese adults. JAMA 282(22): 2131-2135.

44. Pradhan AD, Manson JE, Rifai N, Buring JE, Ridker PM, et al. (2001) Buring JE, Ridker PM C-reactive protein, interleukin 6, and risk of developing type 2 diabetes mellitus. JAMA 286(3): 327-334.

45. Gregor MF, Hotamisligil GS (2011) Inflammatory mechanisms in obesity. Annu Rev Immunol 29: 415-445.

46. Wood IS, De Heredia FP, Wang B, Trayhurn P (2009) Cellular hypoxia and adipose tissue dysfunction in obesity. Proc Nutr Soc 68(4): 370-377.

47. Kern PA, Ranganathan S, Li C, Wood L, Ranganathan G (2001) Adipose tissue tumor necrosis factor and interleukin-6 expression in human obesity and insulin resistance. Am J Physiol Endocrinol Metab 280(5): E745-E751.

48. Klover PJ, Zimmers TA, Koniaris LG, Mooney RA (2003) Chronic exposure to interleukin-6 causes hepatic insulin resistance in mice. Diabetes 52(11): 2784-2789

49. Ridker PM, Rifai N, Stampfer MJ, Hennekens CH (2000) Plasma concentration of interleukin- 6 and the risk of future myocardial infarction among apparently healthy men. Circulation 101(15): 17671772.

50. Wake DJ, Walker BR (2004) 11-beta-Hydroxysteroid dehydrogenase type 1 in obesity and the metabolic syndrome. Mol Cell Endocrinol 215(1-2): 45-54.

51. Masuzaki H, Paterson J, Shinyama H, Morton NM, Mullins JJ, et al (2001) A transgenic model of visceral obesity and the metabolic syndrome. Science 294: 2166-2170.

52. Cho CE, Sánchez HD, Reza López SA, Huot PS, Kim YI, et al. (2013) High folate gestational and post-weaning diets alter hypothalamic feeding pathways by DNA methylation in Wistar rat offspring. Epigenetics 8(7): 710-719.

53. Kelly KB, Kennelly JP, Ordonez M, Nelson R, Leonard K, et al. (2016) Excess folic acid increases lipid storage, weight gain, and adipose tissue inflammation in high fat diet-fed rats. Nutrients 8(10): E594.

54. Bird JK, Ronnenberg AG, Choi SW, Du F, Mason JB, et al. (2015) Obesity is associated with increased red blood cell folate despite lower dietary intakes and serum concentrations. J Nutr 145(1): 79-86.

55. Smith AD, Kim YI, Refsum H (2008) Is folic acid good for everyone? Am J Clin Nutr 87: 517-533.

56. Abelson P, Kennedy D (2004) The obesity epidemic. Science 304(5676): 1413.

57. Kelly T, Yang W, Chen CS, Reynolds K, He J (2008) Global burden of obesity in 2005 and projections to 2030. Int J Obes 32(9): 1431-1437.

58. Prentice A (2006) The emerging epidemic of obesity in developing countries. Int J Epidemiol 35(1): 92-99.

59. Huffman DM, Barzilai N (2010) Contribution of adipose tissue to health span and longevity contribution of adipose tissue to health span and longevity. Body Composition and Aging 37: 1-19.

60. Stefan N, Fritsche A, Schick F (2016) Phenotypes of prediabetes and stratification of cardiometabolic risk. Lancet-Diabetes \& Endocrinol 4(9): 789-798.

61. Roden M, Price TB, Perseghin G, Petersen KF, Rothman DL, et al. (1996) Mechanism of free fatty acid-induced insulin resistance in humans. J Clin Invest 97(12): 2859-2865. 
62. Karpe F, Dickmann JR, Frayn KN (2011) Fatty acids, obesity and insulin resistance: Time for a re-evaluation. Diabetes 60(10): 2441-2449.

63. Marcial JM, Altieri PI, Banchs H, Escobales N, Crespo M (2011) Metabolic syndrome among puerto ricans and other Hispanic populations. P R Health Sci J 30(3): 145-151.

64. Boden G (1996) Fatty acids and insulin resistance. Diabetes Care 19(4): 394-395.

65. Kahn SE (2001) Clinical review 135: the importance of beta-cell failure in the development and progression of type 2 diabetes. J Clin Endocrinol Metab 86(9): 4047-4058.

66. Al-Goblan AS, Al-Alfi MA, Khan MZ (2014) Mechanism linking diabetes mellitus and obesity. Diabetes Metab Syndr Obes 7: 587-591.
67. Kasuga M (2006) Insulin resistance and pancreatic $\boldsymbol{\beta}$ cell failure. J Clin Invest 116(7): 1756-1760.

68. Ershow AG (2009) Environmental influences on development of type 2 diabetes and obesity: challenges in personalizing prevention and management. J Diabetes Sci Technol 3(4): 727-734.

69. Haffner SM, Agostino DR, Mykkänen L, Tracy R, Howard B, et al. (1999) Insulin sensitivity in subjects with type 2 diabetes. Relationship to cardiovascular risk factors: the Insulin Resistance Atherosclerosis Study. Diabetes Care 22(4): 562-568.

70. Wilkin TJ (2001) The accelerator hypothesis: weight gain as the missing link between type I and type II diabetes. Diabetologia 44(7): 914-922.
Creative Commons Attribution 4.0 International License

For possible submissions Click Here
Submit Article

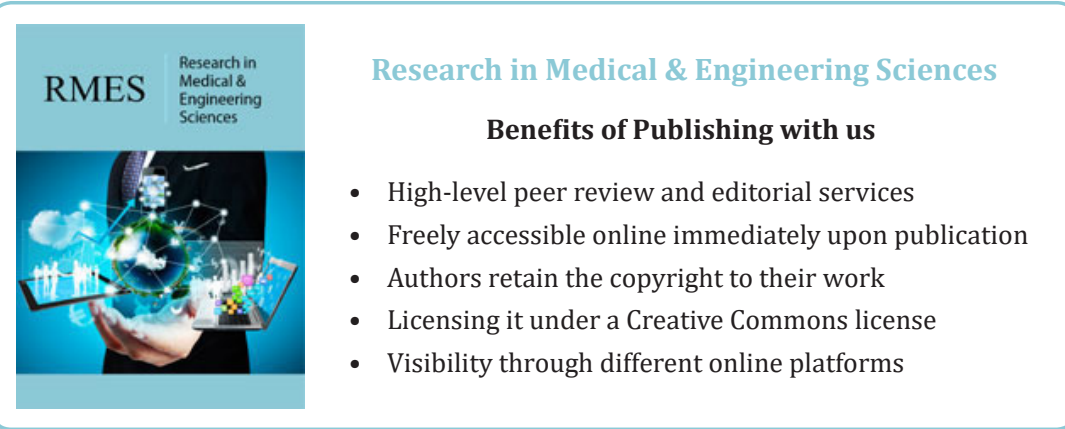

